\title{
Regular and chaotic oscillations of a Timoshenko beam subjected to mechanical and thermal loadings
}

Received: 1 December 2013 / Accepted: 9 August 2014 / Published online: 27 August 2014

(C) The Author(s) 2014. This article is published with open access at Springerlink.com

\begin{abstract}
Dynamics of a Timoshenko beam under an influence of mechanical and thermal loadings is analysed in this paper. Nonlinear geometrical terms and a nonuniform heat distribution are taken into account in the considered model. The mathematical model is represented by a set of partial differential equations (PDEs) which takes into account thermal and mechanical loadings. The problem is simplified to two PDEs and then reduced to ordinary differential equations (ODEs) by means of the Galerkin method taking into account three modes of a linear Timoshenko beam. Correctness of the analytical model is verified by a finite element method. Then, the nonlinear model is studied numerically by a continuation method or by a direct numerical integration of ODEs. An effect of the temperature distribution on the resonance near the first natural frequency and on stability of the solutions is presented. The increase of mechanical loading results in hardening of the resonance curve. Thermal loading may stabilise the beam dynamics when the temperature is decreased. The elevated temperature may transit dynamics from regular to chaotic oscillations.
\end{abstract}

Keywords Timoshenko beam · Thermal loading · Nonlinear vibrations · Nonlinear resonances · Chaos

\section{Introduction}

The beams are fundamental structural elements commonly used in many branches of engineering. They are often applied in order to model rotating blades, for example, blades of a helicopter rotor [1,2] or blades of turbines. Recently, these structures are often made of modern materials e.g. composites which, in spite of the

Communicated by Francesco dell'Isola and Giuseppe Piccardo.

A. Warminska

Department of Thermodynamics, Fluid Mechanics and Aviation Propulsion Systems, Lublin University of Technology,

Nadbystrzycka 36, 20-618 Lublin, Poland

E-mail: a.warminska@pollub.pl

Tel.: +48 815384745

E. Manoach

Institute of Mechanics, Bulgarian Academy of Sciences, Acad. G. Bonchev 4, 1113 Sofia, Bulgaria

E-mail: e.manoach@imbm.bas.bg

Tel.: +35929733140

J. Warminski $(\varangle) \cdot$ S. Samborski

Department of Applied Mechanics, Lublin University of Technology, Nadbystrzycka 36, 20-618 Lublin, Poland

E-mail: j.warminski@pollub.pl

Tel.: +48 815384197

S. Samborski

E-mail: s.samborski@pollub.pl 
fact they are very light, are able to carry large loadings. From the other side, in certain dynamical conditions, they can oscillate with large amplitudes. Moreover, in a real environment, the dynamical response may be essentially changed e.g. by varied temperature. Therefore, for such structures, more adequate mathematical models must be developed to understand better the structure dynamics and phenomena which may occur due to mechanical and thermal loadings.

Linear and nonlinear vibrations of beams have been deeply investigated for many years. In books of Nayfeh and Balachandran [3], Nayfeh and Pai [4], and recently Lacarbonara [5], large vibrations of structures with a geometrical type of nonlinearity have been presented. In such structures stiffness, and consequently, the resonance frequencies and mode shapes are amplitude dependent. The nonlinear vibrations theory has been applied to Euler-Bernoulli model with a large nonlinear curvature and to shear deformable beam models as well. The influence of the nonlinear terms on the bifurcation scenario and possible resonances has been discussed. Structures made of isotropic or anisotropic materials, including composites, have been analysed by mentioned above authors. Recently, Luongo and Zulli [6] published a book providing a complete guidance to the modelling and applications of nonlinear beams and cables. Several models with different complexity, including nonlinear models of elastic and visco-elastic, straight or curved, planar or nonplanar, shearable or unshearable continuous structures, are presented there. The statics and dynamics of prestressed beams which may arise, for example, from thermal loading have also been studied. The so-called general beam theory has been presented in [7] and recently in [8].

The advanced composite beam theory has been presented in [9]. The beam models considered various configurations of lamina with reinforced fibres orientation, closed or open cross-sectional shapes and anisotropy of material. Apart from analytical approach, some authors use the classical FEM [10,11] and semi-analytical methods $[12,13]$ to study nonlinear dynamics of beams with complex geometry. The generalised beam theory developed to investigate mechanics of composite thin-walled structures made of laminates with orthotropic properties is presented in [14]. The fundamental equations based on the first-order beam theory and related boundary conditions are derived, and the physical meaning of the obtained model is clarified. Recently, apart from classical laminated composite beams or plates with straight reinforcing fibres, new composite structures with curvilinear fibres are developed. The review of mechanical properties and modelling of such structures are delivered in [15].

In many papers, the environmental conditions are neglected in the model. One of the essential environmental factors which has to be taken into account is temperature which may vary in high ranges in real mechanical or aerospace applications; therefore, its variations may affect substantially the structure response. Thermal loadings introduce stresses due to thermal expansion, which lead to changes in the modal properties and resonance zones.

The basis of thermo-elastic vibrations was published in books [16-18]. The importance of a heat transfer in rods, beams, and plates was shown there by formulated theory of thermo-elasticity and practical applications, as well. Problems of thermally induced vibrations or thermal buckling have been analysed in $[19,20]$. A sandwich beam with a visco-elastic core has been analysed for buckling and vibration behaviours in thermal environments, using the finite element method. Variation of natural frequencies and the loss factors with respect to temperature have been investigated there. An analytical model for the vibration of horizontal self-weighted and thermally stressed beams with geometrical nonlinearities has been presented in [21]. Results showed that the thermal loads caused by climatic variations have a significant effect on the natural frequencies of slender beams or cables. Under the self-weight, frequencies had a complex thermal behaviour which varied nonlinearly and nonmonotically with respect to the temperature. The prebending phenomenon of thermally prestressed beams affected essentially the vibration modes [22].

In fact, the thermal and stress-strain fields are coupled [18,23]. However, considering that a heat flow is often much slower than related to elasticity mechanical loading, it is reasonable to assume that the temperature distribution is independent of the deformation. This approach is widely used to model the thermo-elastic behaviour of structures. In some cases in order to simplify the model, it is assumed that the structure gets the elevated temperature. This approach has been used to model geometrically nonlinear vibrations of structures [24-26].

Thermo-mechanical, geometrically nonlinear vibrations of plates and beams, correspondingly, are presented in [27] and [28]. Authors demonstrated very reach nonlinear dynamics including, periodic, quasiperiodic and chaotic oscillations. The proposed models have been tested experimentally as well. In [27], temperature has been assumed as linearly distributed along a plate thickness, while in [28] has been uniformly distributed. 
A thermo-mechanical model of a Timoshenko beam subjected to mechanical and thermal loadings, assuming elevated ambient temperature has been proposed in [29]. The set of PDEs has been reduced to the one degree of freedom nonlinear system with thermal components. It has been shown there that the elevated temperature moves the resonance zones. The solutions of the reduced model have been obtained analytically by the multiple timescale method. In order to detect different classes of the primary and secondary, internal or external resonances the multimodal approach are required. The three-mode reduction of the beam's model for thermo-elastic vibration has been presented in [30]. Again, the temperature has been accepted to be uniformly distributed along the beam length and thickness. It has been shown that for large vibrations, apart from the shift of the resonance zones, the elevated temperature introduced instabilities in the solutions, resulting in specific bifurcations and chaotic oscillations. The effect of temperature on selected internal resonances of a homogenous aluminium beam and the shift of the resonance zones are presented also in [31].

The required dimension of the reduced model depends on the type of nonlinearities, external and thermal loadings, boundary conditions, and the structural parameters. Quantitative analysis of various classes of resonances based on algebraic and geometrical methods is presented in papers [32,33]. The nature of possible resonances is discussed in detail pointing the so-called proper and improper resonant terms, depending whether they are or not associated with any resonances.

The temperature distribution plays also very important role in smart structures [34] as it has to be properly taken into account in the applied control strategy.

The purpose of this paper was to study dynamic phenomena of a geometrically nonlinear Timoshenko beam subjected to thermal and mechanical loadings [30]. In this case, however, the temperature is nonuniformly distributed along the beam length and thickness. The assumed nonuniform temperature field results in the initial thermal preloading and related the preset configuration. The assumed temperature distribution has a fundamental meaning for obtained bifurcations, nonperiodic or chaotic oscillations which arise due to the temperature variation.

\section{Timoshenko beam with a heat distribution}

The considered structure is a beam made of elastic composite material subjected to thermal and mechanical loadings. The beam of length $L$, thickness $h$, and width $b$, and the reference coordinate system is presented in Fig. 1. A coordinate set is fixed in the middle of the beam at its left edge (boundary).

Because of the limited applicability of the Euler-Bernoulli beam model, [35-38] the Timoshenkos beam model which is close to the two-dimensional theory, for a case of practical importance is adopted in this paper. Furthermore, the extended version of Timoshenko beam model has been assumed. The model accounts the shear deformation, rotary inertia, and the geometrically nonlinear longitudinal displacements which are a source of nonlinearity.

The extended mathematical model of the Timoshenko beam presented in Fig. 1 has been derived in paper [23]

$$
\begin{aligned}
\frac{c_{p}}{\lambda_{T}} \frac{\partial T}{\partial t} & =\frac{\partial^{2} T}{\partial x^{2}}+\frac{\partial^{2} T}{\partial z^{2}}-\frac{\alpha_{T} E T_{0}}{\lambda_{T}} \frac{\partial \varepsilon}{\partial t} \\
E F \frac{\partial^{2} u}{\partial x^{2}} & =-E F \frac{\partial w}{\partial x} \frac{\partial^{2} w}{\partial x^{2}}+E b \alpha_{T} \frac{\partial \gamma_{T}}{\partial x}
\end{aligned}
$$

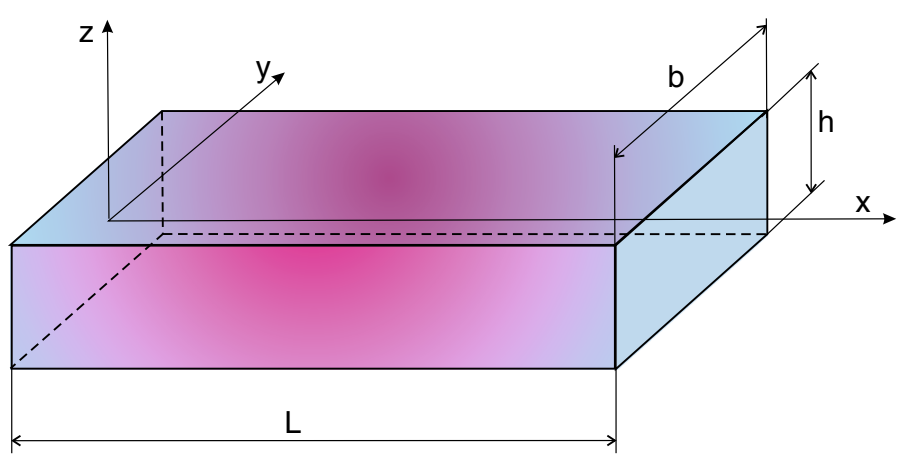

Fig. 1 Schematic beam model with indicated coordinates and dimensions 


$$
\begin{gathered}
E I \frac{\partial^{2} \psi}{\partial x^{2}}+k G F\left(\frac{\partial w}{\partial x}-\psi\right)+\alpha_{T} E b \frac{\partial \chi_{T}}{\partial x}-c_{2} \frac{\partial \psi}{\partial t}-\rho I \frac{\partial^{2} \psi}{\partial t^{2}}=0 \\
k G F\left(\frac{\partial^{2} w}{\partial x^{2}}-\frac{\partial \psi}{\partial x}\right)+E F\left[\frac{\partial u}{\partial x}+\frac{1}{2}\left(\frac{\partial w}{\partial x}\right)^{2}-\alpha_{T} h^{-1} \gamma_{T}\right] \frac{\partial^{2} w}{\partial x^{2}}-c_{1} \frac{\partial w}{\partial t}-\rho F \frac{\partial^{2} w}{\partial t^{2}}=-p(x, t)
\end{gathered}
$$

where $F=b h$ is the area of the beam cross section, $\rho$ material density, $I=b h^{3} / 12, T(x, z, t)$ is the current temperature, $T_{0}$ is the reference constant temperature, $\lambda_{T}$ is the thermal conductivity, and $c_{p}$ is the heat capacity per unit volume. Variables $u(x, t), w(x, t)$ are, respectively, the longitudinal and transverse displacements of the beam, and $\psi(x, t)$ is the rotation of the cross section to the longitudinal axis, $p(x, t)$ is external mechanical loading, and $c_{1}$ and $c_{2}$ represent assumed arbitrary linear damping. $G$ and $E$ are, respectively, shear and Young modulus, and $k$ is the shear correction factor. Functions $\chi_{T}(x, z, t)$ and $\gamma_{T}(x, z, t)$ defined as:

$$
\chi_{T}=\int_{-h / 2}^{h / 2} T(x, z, t) z d z, \quad \gamma_{T}=\int_{-h / 2}^{h / 2} T(x, z, t) d z,
$$

represent bending moment and longitudinal force which arise due to temperature changes.

In the studied model, we assume that a cross section is treated as a local rigid body and it remains plane after deformation (the warping effect is neglected). According to the Timoshenko beam theory, the effect of shear distortion and rotary inertia is taken into account [35]. Thus, motion of the beam cross section depends on two independent variables transverse displacement $w$ and the angle of the rotation of the cross section $\psi$. The total angle of rotation is a sum of the angle of rotation of the cross section due to the bending moment and the angle of distortion due to shear. The inertia of the rotation due to bending is also included in the Timoshenko beam model, while the shear inertia is neglected in Eq. (1c). Furthermore, the influence of the longitudinal inertia is neglected as well in Eq. (1b). This assumption is accepted for transverse vibrations of beams by many authors $[12,13,35,39]$. We also assume that the material properties are independent of the temperature field, i.e. temperature varies in a range which does not influence the material properties.

The model includes linear curvature defined as $\kappa=-\frac{\partial \psi}{\partial x}$ and large displacement field composed of nonlinear mechanical and linear thermal loadings

$$
\varepsilon=-z \frac{\partial \psi}{\partial x}+\alpha_{T}\left(T-T_{0}\right)+\frac{\partial u}{\partial x}+\frac{1}{2}\left(\frac{\partial w}{\partial x}\right)^{2}
$$

The heat flow that acts on beam is much slower than the mechanical stress-strain variations. Therefore, we can also assume that the temperature distribution is independent of the deformation. Thus, the thermal field can be defined by a given function which represents a quasi-steady state of the conductivity equation Eq. (1a) for assumed boundary conditions. This allows a reduction of the conductivity equation.

Considering mentioned above assumption $[23,26,29,30]$, and then introducing the dimensionless coordinates and coefficients, defined as:

$\tilde{w}=w / L$, dimensionless displacement of the beam expressed with respect to the beam's length $L$, where $w$ is the displacement in physical units,

$\tilde{x}=x / L, \tilde{z}=z / L$, dimensionless space coordinates where $x, z$ are coordinates in physical units,

$\tilde{t}=t c / L$, dimensionless time, where $c=\sqrt{E / \rho}$,

$\tilde{\omega_{n}}=\omega_{n} L / c$, 一natural dimensionless frequencies, where $\omega_{n}$ is physical frequency in $\mathrm{rad} / \mathrm{s}, n=1,2,3$.

we get the dimensionless equations of motion in the form:

$$
\begin{aligned}
\frac{\partial^{2} u}{\partial x^{2}} & =G_{u}+G_{u}^{T} \\
\frac{\partial^{2} \psi}{\partial x^{2}}+\beta \alpha\left(\frac{\partial w}{\partial x}-\psi\right)-d_{2} \frac{\partial \psi}{\partial t}-\frac{\partial^{2} \psi}{\partial t^{2}} & =G_{1}^{T} \\
\beta\left(\frac{\partial^{2} w}{\partial x^{2}}-\frac{\partial \psi}{\partial x}\right)-d_{1} \frac{\partial w}{\partial t}-\frac{\partial^{2} w}{\partial t^{2}} & =-p+G_{2}^{L}+G_{2}^{T}
\end{aligned}
$$


(a)

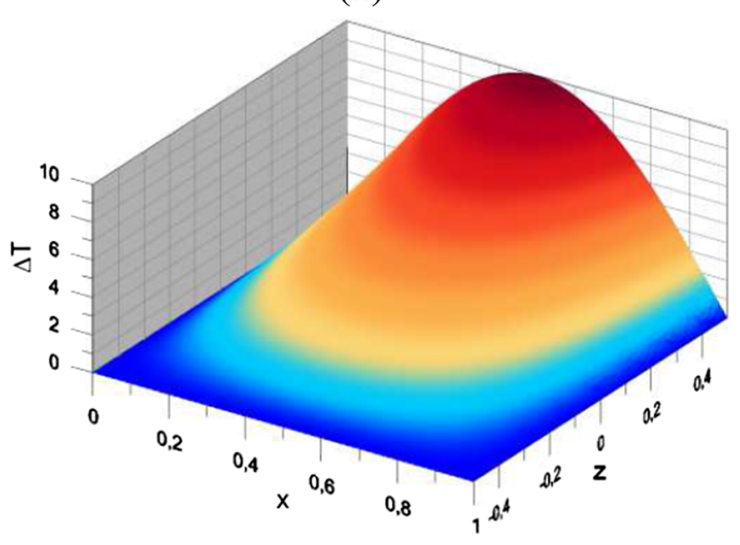

(b)

(c)
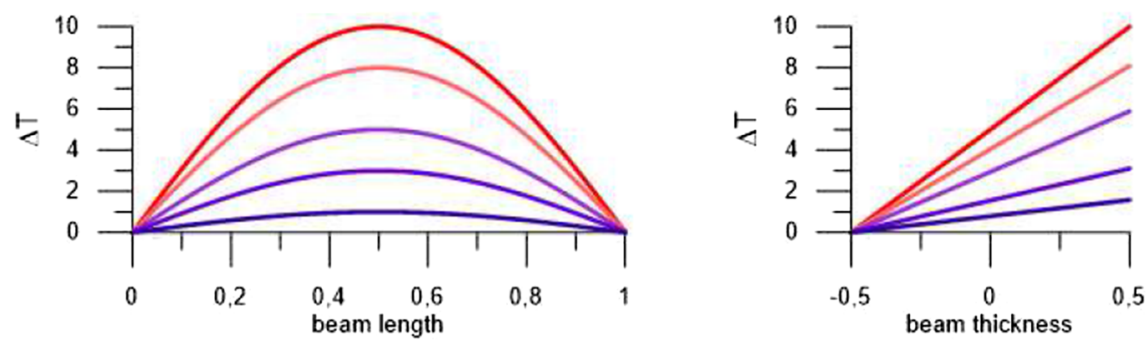

Fig. 2 Temperature distribution, 3D plot (a); distributions along beam length (b) and thickness (c)

where $\alpha=12 L^{2} / h^{2}, \beta=k G / E$. Mechanical loading $p=p(x, t)$ is distributed along $x$ axis, it is a function of space and time, and $d_{1}, d_{2}$ are the dimensionless coefficients of linear damping included in the model. In the above notation, 'tilde' has been dropped for simplicity. The terms $G_{u}, G_{u}^{T}, G_{1}^{T}, G_{2}^{L}, G_{2}^{T}$ on the right sides of Eq. (4) are defined as:

$$
\begin{array}{r}
G_{u}=-\frac{\partial w}{\partial x} \frac{\partial^{2} w}{\partial x^{2}}, \quad G_{u}^{T}=\alpha_{T} T_{0} \frac{\partial \gamma_{\theta}}{\partial x}, \quad G_{1}^{T}=\alpha_{T} T_{0} \frac{12 L}{h} \frac{\partial \chi_{\theta}}{\partial x} \\
G_{2}^{L}=-\left[\frac{\partial u}{\partial x}+\frac{1}{2}\left(\frac{\partial w}{\partial x}\right)^{2}\right] \frac{\partial^{2} w}{\partial x^{2}}, \quad G_{2}^{T}=\alpha_{T} T_{0} \gamma_{\theta} \frac{\partial^{2} w}{\partial x^{2}},
\end{array}
$$

and

$$
\chi_{\theta}=\int_{-1 / 2}^{1 / 2} \theta(x, z, t) z d z, \quad \gamma_{\theta}=\int_{-1 / 2}^{1 / 2} \theta(x, z, t) d z .
$$

Functions $\chi_{\theta}(x, z, t)$ and $\gamma_{\theta}(x, z, t)$ represent additional bending moment and longitudinal force (dimensionless) which arise due to the temperature changes. These functions depend on $\theta(x, z, t)$ which is the dimensionless heat distribution in space, along beam length and thickness, and in time domain.

In contrast to papers $[29,30]$ the distribution of temperature along $x$ and $z$ axis is not constant but assumed by the function

$$
\theta(z, x, t)=\Theta \sin \pi x\left(z+\frac{1}{2}\right)
$$

where $\Theta$ is temperature amplitude. It is accepted that the beam is in a temperature environment which imposes a linear distribution along the thickness of the beam, a constant distribution along the width of the beam, and sine distribution along the length. The temperature distribution in 3D plot and 2D cross sections is presented in Fig. 2.

The assumed temperature function may represent, for example, a heat source located above the middle of the beam. Then, the left and right boundaries of the beam are "cold" (blue colour) and in the middle, the beam 
is "hot". We may say that at $x=0, x=L$ and $z=-h / 2$, the beam is heat insulated. The distribution along the thickness is linear with a "hot" upper and "cold" bottom wall. The function $\theta$ is a difference between the current and the initial temperature.

After integration, from (6), we obtain the components $\chi_{\theta}, \gamma_{\theta}$

$$
\chi_{\theta}=\frac{\Theta}{12} \sin \pi x, \quad \gamma_{\theta}=\frac{\Theta}{2} \sin \pi x .
$$

and consequently, terms related to thermal expansion

$$
\begin{aligned}
G_{u}^{T} & =\alpha_{T} T_{0} \Theta \frac{\pi}{2} \cos \pi x, \\
G_{1}^{T} & =\alpha_{T} T_{0} \Theta \frac{\pi L}{h} \cos \pi x, \\
G_{2}^{T} & =\frac{1}{2} \alpha_{T} T_{0} \Theta \sin \pi x \frac{\partial^{2} w}{\partial x^{2}} .
\end{aligned}
$$

Note that $G_{1}^{T}$ term has to be rescaled considering both dimensionless coordinates in $x$ and $z$ directions. (4)

Denoting $G_{u}^{A}=G_{u}+G_{u}^{T}$, the longitudinal displacement can be found from the first equation of the set

$$
\begin{aligned}
\frac{d u}{d x} & =K+\int_{0}^{x} G_{u}^{A}(\xi) d \xi, \\
u(x) & =x\left[K+\int_{0}^{x} G_{u}^{A}(\xi) d \xi\right]-\int_{0}^{x} \xi G_{u}^{A}(\xi) d \xi
\end{aligned}
$$

Detailed calculations of the integrals and constant $K$ are presented in "Appendix A". Having defined the constant $K$, we get

$$
G_{2}^{L}(x, t)=-\left[\int_{0}^{1}\left(\frac{\partial w}{\partial \xi}\right)^{2} d \xi-T_{0} \Theta \alpha_{T}\left(\frac{1}{\pi}-\frac{1}{2} \sin \pi x\right)\right] \frac{\partial^{2} w}{\partial x^{2}} .
$$

Now the model can be simplified to two partial differential equations (PDEs) which have the dimensionless form:

$$
\begin{aligned}
\frac{\partial^{2} \psi}{\partial x^{2}}+\beta \alpha\left(\frac{\partial w}{\partial x}-\psi\right)-d_{2} \frac{\partial \psi}{\partial t}-\frac{\partial^{2} \psi}{\partial t^{2}} & =G_{1}^{T} \\
\beta\left(\frac{\partial^{2} w}{\partial x^{2}}-\frac{\partial \psi}{\partial x}\right)-d_{1} \frac{\partial w}{\partial t}-\frac{\partial^{2} w}{\partial t^{2}} & =-p+G_{2}^{T}+G_{2}^{L} .
\end{aligned}
$$

As we can see the left side of the equations describe classical Timoshenko beam model, while on the right side, there are additional terms due to geometric nonlinearity, thermal and external loadings.

\section{Modal reduction}

The model of a beam has been reduced from PDEs to ordinary differential equations (ODEs) by means of Galerkin's orthogonalisation method. The generalised displacements vector is assumed in a series of a product of the quasi-normal modes (shape functions) $w_{n}(x), \psi_{n}(x)$ and generalised, time-dependent coordinates $q_{n}(t)$ :

$$
\begin{aligned}
& w(x, t)=\sum_{n=1}^{N_{f}} w_{n}(x) q_{n}(t) \\
& \psi(x, t)=\sum_{n=1}^{N_{f}} \psi_{n}(x) q_{n}(t)
\end{aligned}
$$


$N_{f}$ is a number of assumed modes. According to the Galerkin's procedure, the quasi-normal modes should satisfy geometrical and dynamical (natural) boundary conditions. For the needs of the reduction procedure, we take linear modes of a simply supported beam $(S S-S S)$. The eigenvalues and eigenmodes have been presented in detail in the papers $[29,30]$. Considering that $w_{n}$ and $\psi_{n}$ are solutions of the eigenvalue problem, we substitute (13) into (12). Then, multiplying (12a) by $\psi_{m}$, and (12b) by $w_{m}$, summing up both equations, integrating over the beam length and invoking the orthogonality condition, we get

$$
\begin{aligned}
& \ddot{q}_{n}(t)+2 \xi_{n} \omega_{n} \dot{q}(t)+\omega_{n}^{2} q_{n}(t) \\
& \quad=\int_{0}^{1} p(x, t) w_{n}(x) d x-\int_{0}^{1} G_{1}^{T}(x) \psi_{n}(x) d x-\int_{0}^{1} G_{2}^{L}(x, t) w_{n}(x) d x-\int_{0}^{1} G_{2}^{T}(x, t) w_{n}(x) d x
\end{aligned}
$$

In the above equations, $\omega_{n}$ is the $n$-th natural frequency of the linear undamped Timoshenko beam and $\xi_{n}$ is a modal damping coefficient. Note that proportional modal damping has been accepted in the considered model. Details of the applied Galerkin procedure are presented in "Appendix B".

In order to have comparison with the results presented in [30], we consider the first three modes of the expansion, i.e. $N_{f}=3$. Because the main analysis will be performed around the main resonance zone near the first natural frequency and the thermal and mechanical loadings are distributed according to this natural mode, the influence of higher modes is hardly observed. The three-mode reduction is a good approximation in this case. A set of three nonlinear ODEs of motion takes the form:

$$
\begin{aligned}
\ddot{q}_{1} & +2 \xi_{1} \omega_{1} \dot{q}_{1}+\omega_{1}^{2} q_{1}+C_{1,111} q_{1}^{3}+C_{1,122} q_{1} q_{2}^{2}+C_{1,123} q_{1} q_{2} q_{3}+C_{1,133} q_{1} q_{3}^{2} \\
& +C_{1,112} q_{1}^{2} q_{2}+C_{1,113} q_{1}^{2} q_{3}+C_{1,0}^{T} \Delta T+C_{1}^{T} \Delta T q_{1}=p_{1} \sin \Omega t \\
\ddot{q}_{2} & +2 \xi_{2} \omega_{2} \dot{q}_{2}+\omega_{2}^{2} q_{2}+C_{2,222} q_{2}^{3}+C_{2,211} q_{2} q_{1}^{2}+C_{2,123} q_{1} q_{2} q_{3}+C_{2,233} q_{2} q_{3}^{2} \\
& +C_{2,122} q_{1} q_{2}^{2}+C_{2,223} q_{2}^{2} q_{3}+C_{2}^{T} \Delta T q_{2}=p_{2} \sin \Omega t \\
\ddot{q}_{3} & +2 \xi_{3} \omega_{3} \dot{q}_{3}+\omega_{3}^{2} q_{3}+C_{3,333} q_{3}^{3}+C_{3,311} q_{3} q_{1}^{2}+C_{3,123} q_{1} q_{2} q_{3}+C_{3,322} q_{3} q_{2}^{2} \\
& +C_{3,133} q_{1} q_{3}^{2}+C_{3,233} q_{2} q_{3}^{2}+C_{3}^{T} \Delta T q_{3}=p_{3} \sin \Omega t
\end{aligned}
$$

The values of coefficients of Eq. (15) are reported in "Appendix C" for a case of a symmetric cross-ply laminated composite beam. The parameters $p_{1}, p_{2}, p_{3}, \Omega$ are, respectively, amplitudes and frequency of external loading, and $\Delta T=T_{0} \Theta$ is the temperature difference.

\section{Numerical data and FEM model verification}

Numerical calculations have been carried out for data presented in "Appendix C" for a symmetric cross-ply laminated 20 layers composite beam. In order to validate correctness of the theoretical analysis, a 3D beam model in FE Abaqus package was prepared. The FE model consisted of thermally coupled 8-node C3D8T quadratic brick elements with the full integration and hourglassing control. The mesh convergence study was performed having in target reaching as small deviation of the eigenfrequencies, as possible, taking into account the prospective temperature field application, what demanded model simplicity due to its appropriate computational efficiency. The 3D beam's end faces were restrained against warping, in order to keep the model as similar as possible to the simple $1 \mathrm{D}$ one. All the simulations were performed within a geometrically linear regime. The eigenfrequencies and modes of the beam having dimensions $500 \times 5 \times 5 \mathrm{~mm}$ and material data as reported in "Appendix C" were obtained for the first three modes and then compared with theoretical analysis. The results are presented in Table 1.

As we may see in Table 1, the frequencies obtained theoretically from the reduces model are in a good agreement with the FE method. The first three FE eigenmodes without any temperature influence are shown in Table 1, as well.

Then, the space temperature distribution as presented in Fig. 2 has been introduced into FEM by the following function: $T(x, z)=10 \sin (\pi x)(z+0.5)$, where $x$ and $z$ were nondimensional space variables defined in the beams local coordinate system as $x=X / L$ and $z=Z / h$. The calculated deflection of the beams middle point due to the thermal expansion under the above given field was $0.656 \mathrm{~mm}$ (Fig. 3). The thermal deflection of the equivalent analytical model after rescaling was $0.639 \mathrm{~mm}$. Again both models were in a good agreement. We may recall that the assumed temperature distribution may correspond to a heat source 
Table 1 Comparison of natural frequencies from FEM and theoretical model for $\Delta T=0$

\begin{tabular}{|c|c|c|}
\hline Frequency & FEM model (Hz) & $\begin{array}{l}\text { Analytical model } \\
(\mathrm{Hz})\end{array}$ \\
\hline$\omega_{1}$ & 41.08 & 40.98 \\
\hline$\omega_{2}$ & 164.22 & 163.85 \\
\hline$\omega_{3}$ & 369.20 & 368.36 \\
\hline & 0.656 & \\
\hline & 0.437 & \\
\hline
\end{tabular}

Fig. 3 Beam deflection for assumed temperature distribution and $\Delta T=10$

placed above the beam. In such a case, heat is nonuniformly distributed along the beam length and thickness. This distribution caused thermal deflection (buckling) of the beam in the direction of the heat source. Next, the eigenfrequencies for the 3D model were found providing that the beam was still exposed to the temperature field. The results showed a slight decrease of eigenfrequencies as the beam was heated. Namely, the first three frequencies were $38.70,156.30$, and $361.41 \mathrm{~Hz}$, respectively. This result also confirmed those obtained from the analytical model. Moreover, to check the correctness of the reduced analytical model, also static tests under mechanical loading were performed. As regards the dynamical case of FEM, due to very long computation time, the numerical simulations were performed only for one selected dynamical loading with frequency equal to the first natural frequency of the model. Again the result was in accordance with the model described by Eq. (15). The FEM was useful for the validation of the analytical approach, however, was not effective for a study of complex nonlinear phenomena of the considered thermo-mechanical structure. Therefore, in next part for complex dynamics, only the analytical model is studied in detail for small and large vibrations amplitude and parameters varied in a wide range.

\section{Analysis of nonlinear dynamical phenomena under mechanical and thermal loading}

The proposed analytical model (15) allows parametric study of the considered structure. In this paper, we concentrate on structure dynamics near the first resonance zone, i.e. we assume that the excitation frequency $\Omega$ is close to the first natural frequency $\omega_{1}$. Therefore, in (15), we substitute $p_{2}=0, p_{3}=0$. Amplitude and frequency of external loading are varied in order to demonstrate essential bifurcation points or nonlinear phenomena around this resonance zone.

Let us consider first that thermal loading is excluded. We assume that $\Delta T=0$. In such a case, we obtain classical resonance curves due to mechanical loading, only. The resonance curves are computed from Eq. (15) by continuation method [40]. The curves are plotted for temperature $\Delta T=0$ and three selected levels of excitation $p_{1}=10^{-7}, p_{1}=10^{-6}, p_{1}=5 \times 10^{-6}$. The increased amplitude of mechanical excitation results in large oscillations exhibiting stiffening phenomenon. The oscillations are periodic and symmetric with respect to the zero equilibrium position. The resonance curves are presented in Fig. 4a. Now we introduce small thermal loading imposing temperature difference $\Delta T=10$ and with the assumed distribution given by (7). The introduced additional thermal loading is observable by a slight shift of the resonance curves into a lower frequency direction. The time histories of oscillations are slightly shifted from the centre (zero position) into positive direction. This result is in a good agreement with FE model presented in Sect. 4. Furthermore, due to increased temperature, additional small picks near frequency $\Omega \approx 0.01-0.015$, for the largest resonance 
(a)

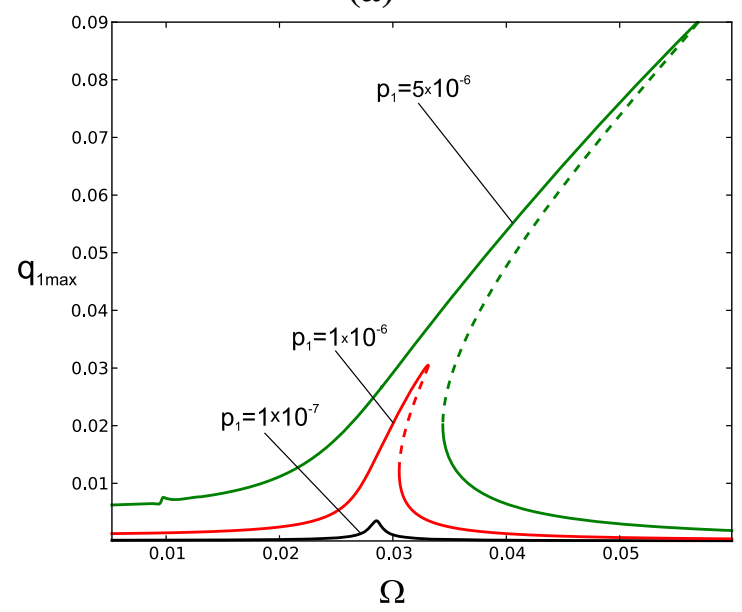

(b)

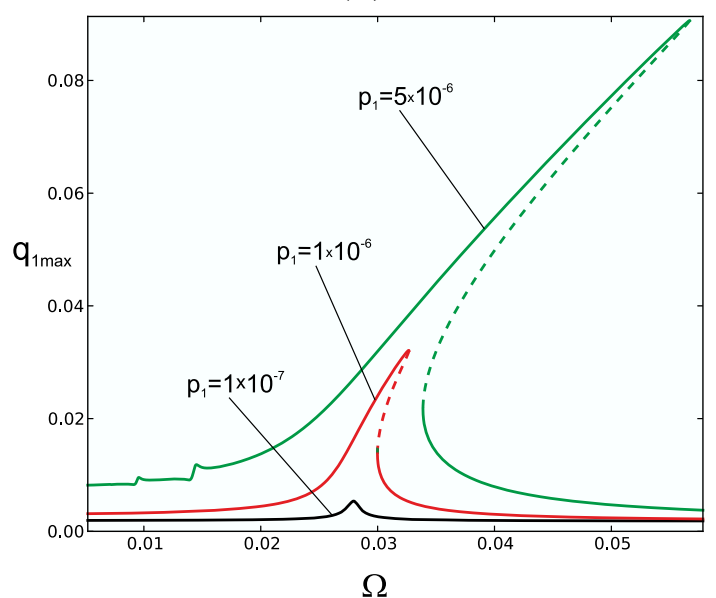

Fig. 4 Resonance curves near the first resonance zone for selected amplitudes of excitation $p_{1}=1 \times 10^{-7}, p_{1}=1 \times 10^{-6}, p_{1}=$ $5 \times 10^{-6} ; \mathbf{a} \Delta T=0, \mathbf{b} \Delta T=10$

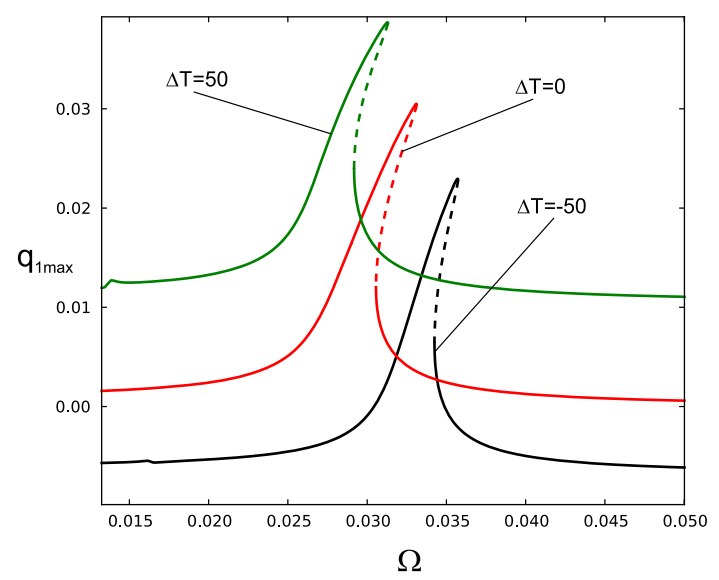

Fig. 5 Resonance curves near the first resonance zone for $\Delta T=50, \Delta T=0, \Delta T=-50$ and excitation $p_{1}=1 \times 10^{-6}$

curve (Fig. 4b), occur. Those picks correspond to the secondary resonances which appear only if the system is nonlinear and both mechanical and thermal loading are large enough to exhibit this phenomenon.

In order to show the thermal loading influence, we consider larger temperature difference $\Delta T=50, \Delta T=$ $0, \Delta T=-50$. In this case, the difference in the resonance curves caused by the influence of thermal loading is clearly visible. We see that for $\Delta T=50$, the resonance curve is shifted to the lower frequencies and amplitudes are moved up (Fig. 5), comparing to the neutral curve for $\Delta T=0$. The negative temperature $\Delta T=-50$ caused a shift of the curve into higher frequencies and a shift of amplitudes below zero.

To present the temperature influence, we have selected and fixed excitation frequency near the first natural mode $\Omega=0.029$. The three levels of excitation amplitude are considered: $p_{1}=1 \times 10^{-7}, p_{1}=1 \times 10^{-6}$ and $p_{1}=5 \times 10^{-6}$. In the case of lowest level of excitation, the beam shows almost linear behaviour, while for two other cases strong nonlinearity is demonstrated (see curves in Fig. 6). The curve for the lowest mechanical loading is almost linear (black curve) with only two small picks on the curve. The elevated temperature increases the level of amplitude. When oscillations due to mechanical loading are large (curves red and green), then thermal loading changes (disturbers) the curve course. In the intervals: $\Delta T \in(75,100)$ for $p_{1}=1 \times 10^{-6}$ and $\Delta T \in(100,125)$ for $p_{1}=5 \times 10^{-6}$, a decrease of amplitude is observed.

Of course for large oscillations and additional thermal loading, we may expect specific bifurcation scenarios. As we mentioned earlier, the force acts with the frequency close to the first natural frequency $\Omega \approx \omega_{1}$. We start possible bifurcation analysis due to mechanical loading by imposing $\Delta T=0$, fixed frequency $\Omega=0.029$, and increasing amplitude of external force $p_{1}$ treated as a bifurcation parameter (Fig. 7a). We notice that in 


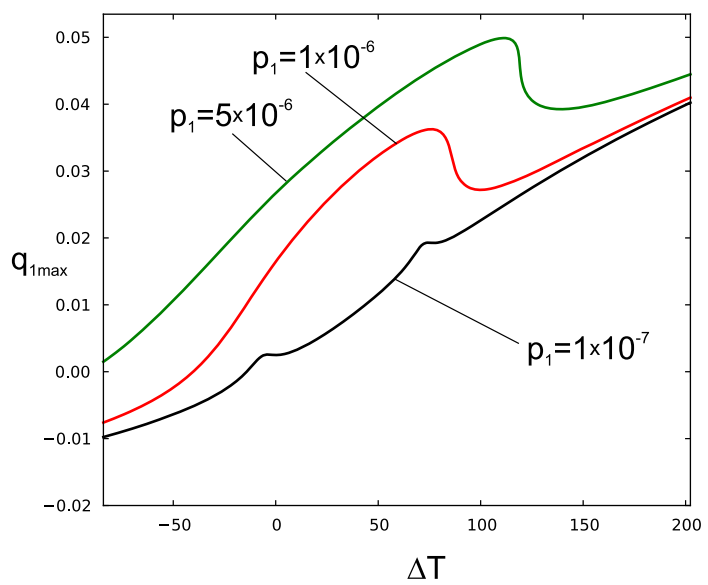

Fig. 6 Influence of thermal loading near the first resonance zone; amplitude against temperature $\Delta T$ for amplitudes of excitation $p_{1}=1 \times 10^{-7}, p_{1}=1 \times 10^{-6}, p_{1}=5 \times 10^{-6}$ and fixed frequency $\Omega=0.029$

(a)

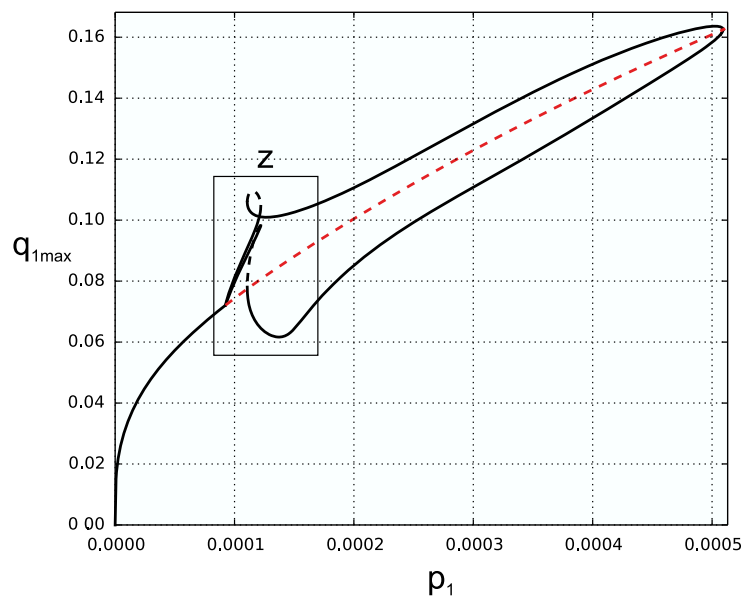

(b)

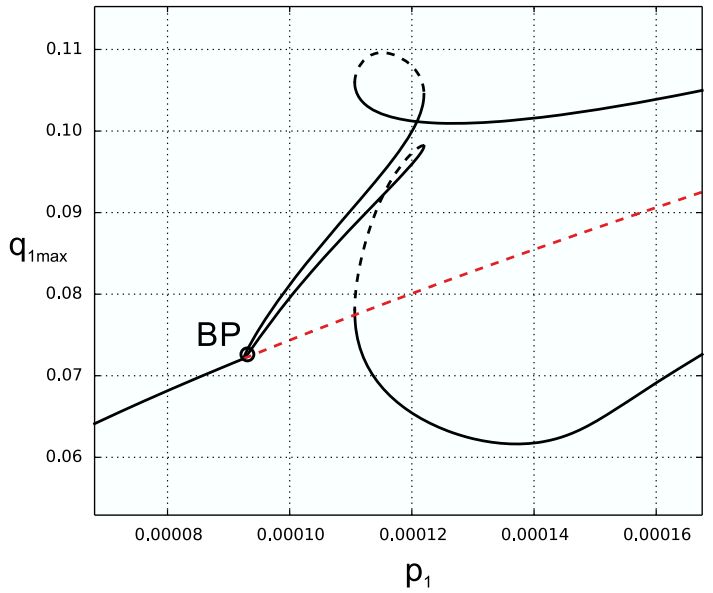

Fig. 7 Bifurcation diagram versus amplitude of mechanical loading $p_{1}$ for $\Omega=0.029, \Delta T=0$; a full curve, $\mathbf{b}$ a zoom of the zone $z$

fact, an increase of mechanical loading increases oscillations amplitude; however, about $p_{1}=9 \times 10^{-5}$ the stable solution looses its stability (red dashed line) and a new stable branch arises, there is a bifurcation point in which a new stable branch arises. This new branch, arising from the branch point $(B P)$, makes a loop around unstable solutions (till about $p_{1}=5 \times 10^{-4}$ ) and returns to $B P$. The solutions around $B P$ are presented in zoom in (Fig. 7b). Time histories for characteristic values of $p_{1}$ before and after $B P$ exhibit qualitative changes in the beam's response. In Fig. 8a, we see harmonic oscillations for $p_{1}=5 \times 10^{-5}$, before the bifurcation point. After $B P$ point, the solution for $p_{1}=1.1 \times 10^{-4}$, laying on the new stable branch, changes qualitatively. Nevertheless, it is still periodic, but composed of more harmonics (Fig. 8b). For $p_{1}=1.2 \times 10^{-4}$, two periodic solutions exist: for lower and upper branch. They are presented, respectively, in Fig. 8c, d. All the mentioned solutions have a period corresponding to the excitation period. After $B P$ point, the solution is still periodic, but it is not furthermore just a single harmonic.

We may expect that if thermal loading is imposed, then the bifurcation scenario may be changed and the selected bifurcation points may be hidden or transformed into new. Let us consider that the temperature drops down. In Fig. 9a, for $\Delta T=-20$ in fact, the bifurcation point $B P$ vanished and there is a small loop on the curve instead. The tendency is similar for $\Delta T=-50$ (Fig. 9b). The unstable part of the solution (dashed line) is observed but for very large amplitude of excitation.

The dynamics changes essentially if the temperature elevates. The bifurcation scenario in this case is shown in Fig. 10 for relatively high temperature $\Delta T=50$, in order to expose qualitative changes. First of 
(a)

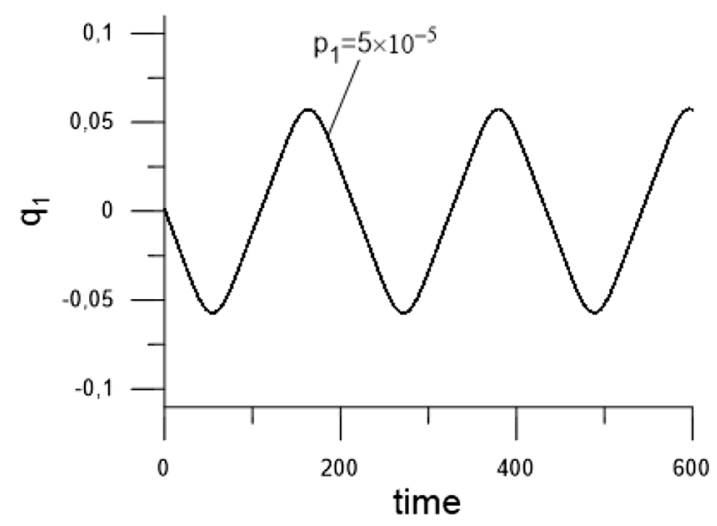

(c)

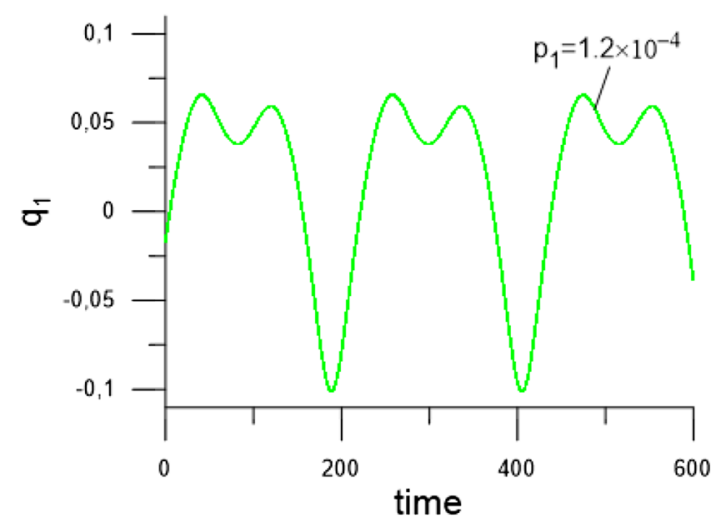

(b)

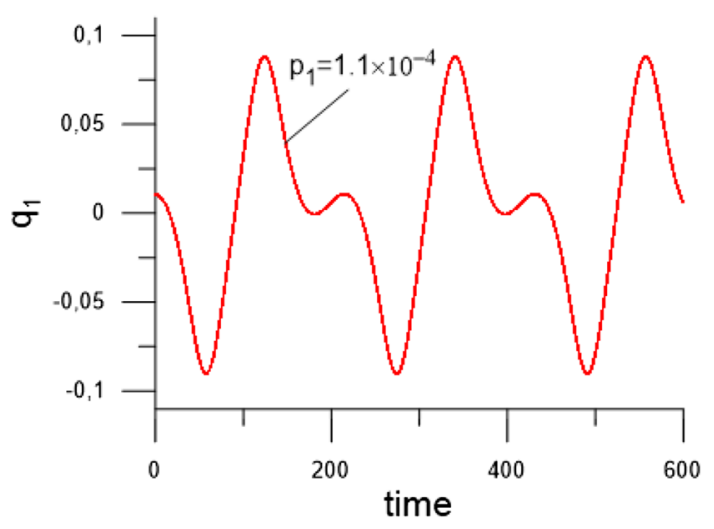

(d)

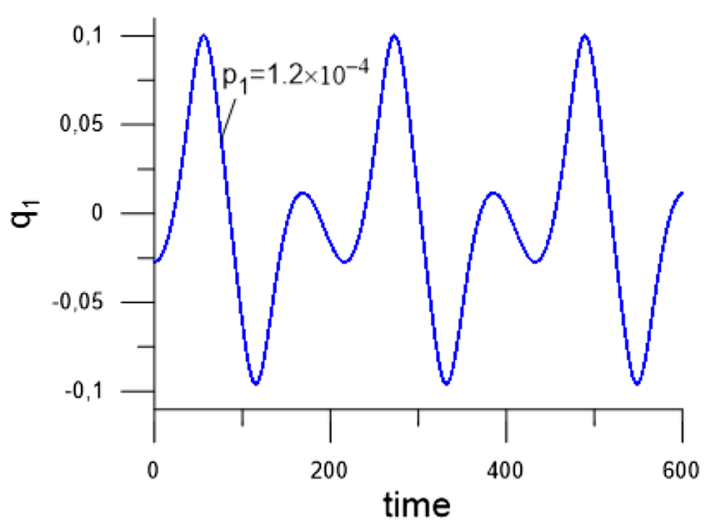

Fig. 8 Time histories for fixed frequency $\Omega=0.029$, temperature $\Delta T=0$ and mechanical loading a $p_{1}=5 \times 10^{-5}$, b $p_{1}=1.1 \times 10^{-4}$, c $p_{1}=1.2 \times 10^{-4}$ (lower branch), $\mathbf{d} p_{1}=1.2 \times 10^{-4}$ (upper branch)

(a)

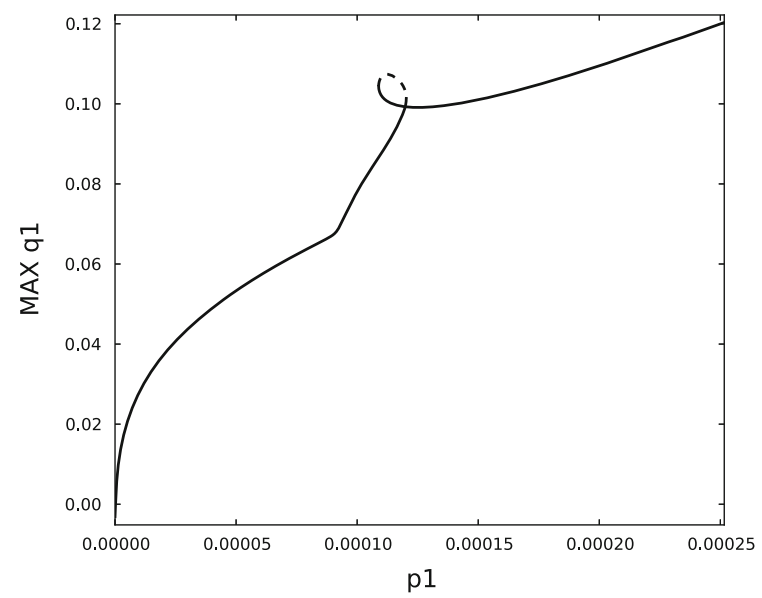

(b)

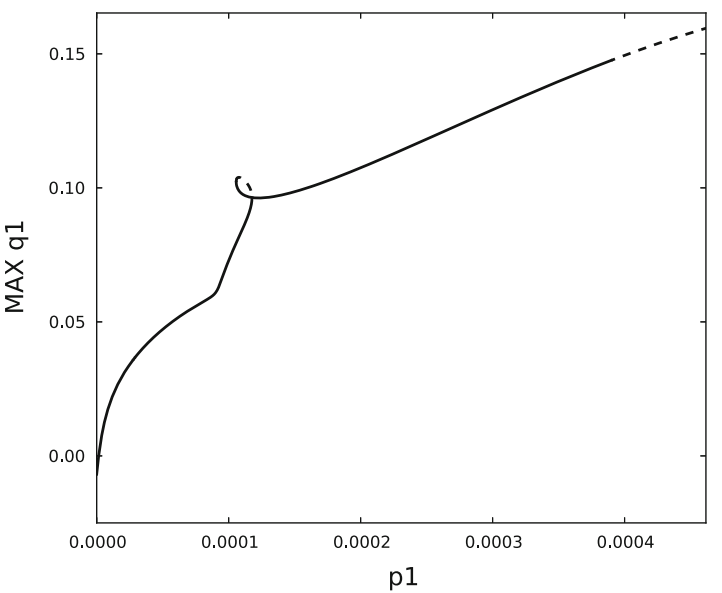

Fig. 9 Bifurcation diagrams versus amplitude of mechanical loading $p_{1}$ near the first resonance zone for temperature $\mathbf{a} \Delta T=-20$, b $\Delta T=-50$ and fixed frequency $\Omega=0.029$ 
(a)

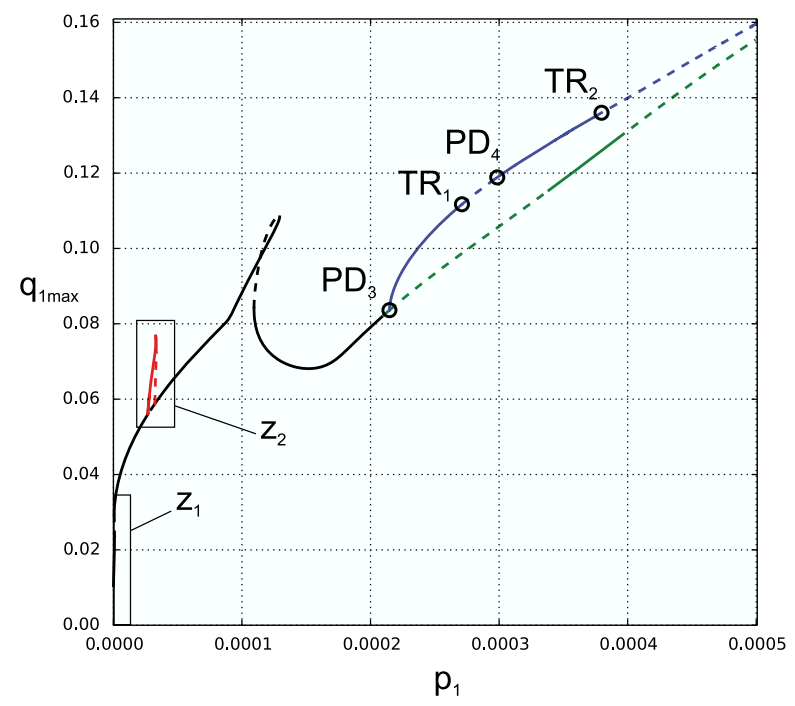

(b)

(c)
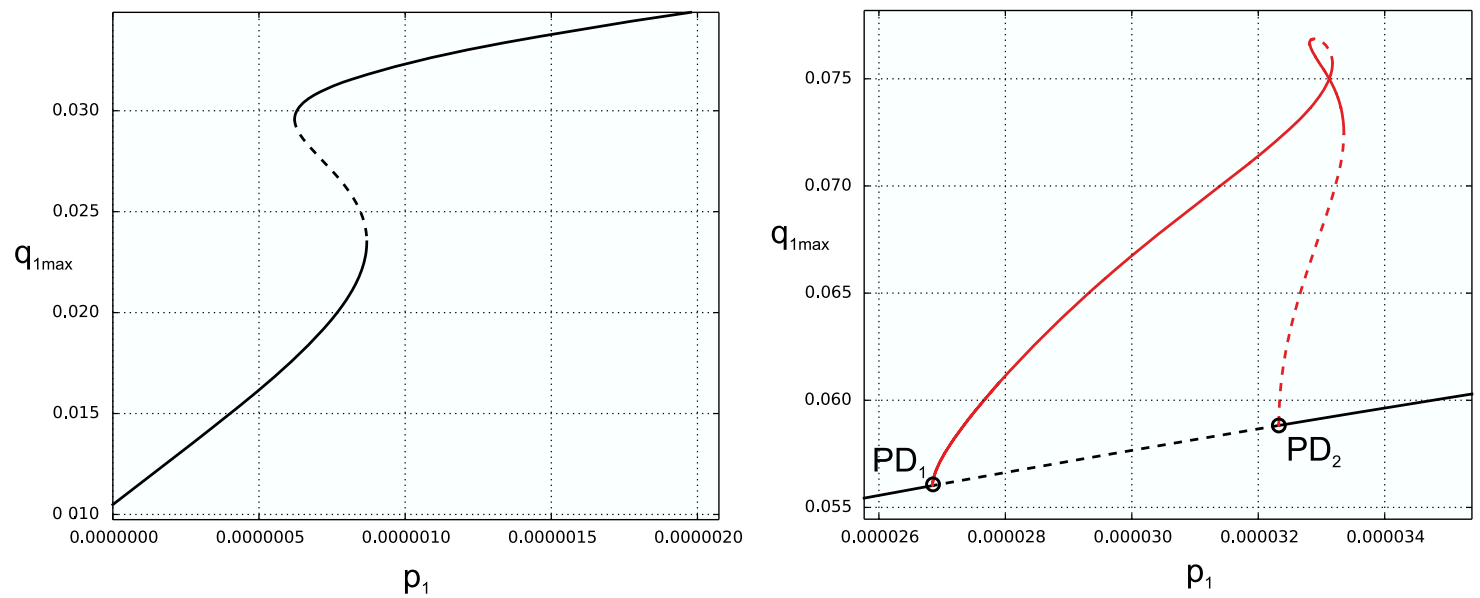

Fig. 10 Bifurcation diagrams versus amplitude of mechanical loading $p_{1}$ near the first resonance zone (a) for temperature $\Delta T=50$ and fixed frequency $\Omega=0.029$, zoom of the zone $z_{1}(\mathbf{b})$ and $z_{2}(\mathbf{c})$

all, the bifurcation point observable for $\Delta T=0$ disappeared. The course of the curve is smooth in this point, and therefore, there is no branch point $B P$ visible in Fig. 7. Around $p_{1}=1 \times 10^{4}$, the curve is smooth. Numerical tests show that the $B P$ exists only if $\Delta T=0$. Small variation in the temperature, positive or negative, eliminates the branch point $B P$. Detailed analysis of the bifurcation diagram Fig. 10 shows that for low amplitude of mechanical loading, the increased temperature makes a fold of the curve, and an narrow unstable part occurs. This is presented as zoom $z_{1}$ in Fig. 10b. Then, for relatively low mechanical loading about $p_{1}=3 \times 10^{-5}$, the main branch becomes unstable and a new branch arises. But in this case, the new solutions start from period doubling bifurcation points $P D_{1}$ and $P D_{2}$ (see zoom $z_{2}$ in Fig. 10c). The period doubling bifurcations are obtained by computing Floquet multiplier [40] starting from the initially computed orbit of period $T$, and then varying the bifurcation parameter, the solution of period $2 T$ has been obtained. The time history corresponding to the period doubling branch is presented in Fig. 11b. This is period $2 T$ solution comparing with those before $P D_{1}$ (Fig. 11a) or after $P D_{2}$ (Fig. 11c) bifurcation points. Another period doubling $P D_{3}$ visible in Fig. 10a arises around parameter $p_{1}=2.2 \times 10^{4}$, then following new branch we get torus bifurcation $T R_{1}$, after which we return to period doubling $P D_{4}$ and then to torus $T R_{2}$. We may expect that in those intervals, the system response is complex. 
(a)

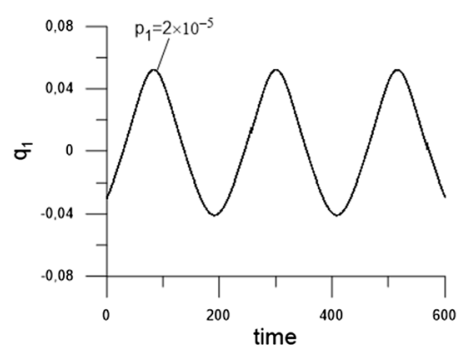

(d)

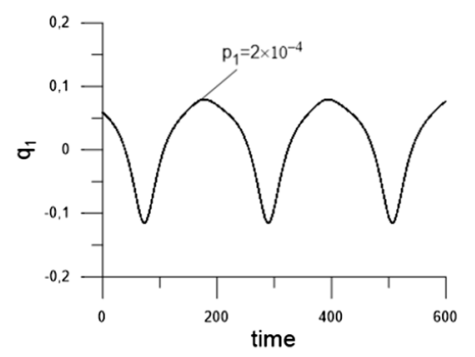

(b)

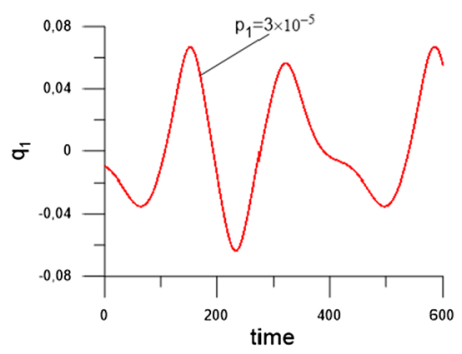

(e)

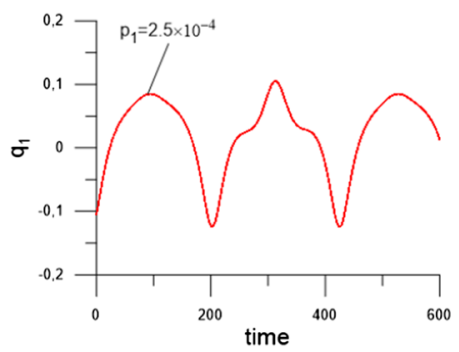

(c)

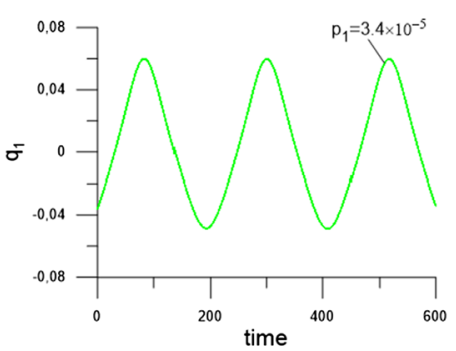

(f)

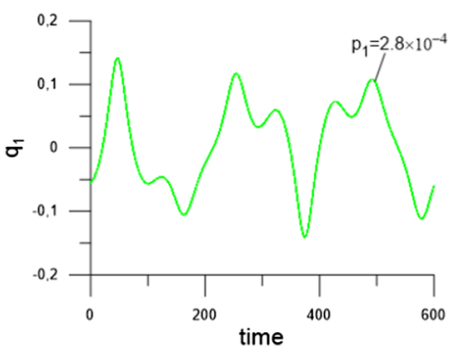

Fig. 11 Time histories for selected values of mechanical loading $p_{1}$ and fixed temperature $\Delta T=50$ and frequency $\Omega=0.029$, for $p_{1}=2 \times 10^{-5}(\mathbf{a}), p_{1}=3 \times 10^{-5}(\mathbf{b}), p_{1}=3.4 \times 10^{-5}(\mathbf{c})$-corresponding to area $z_{2}$ in Fig. 10; for $p_{1}=2 \times 10^{-4}(\mathbf{d})$, $p_{1}=2.5 \times 10^{-5}(\mathbf{e}), p_{1}=2.8 \times 10^{-5}(\mathbf{f})$, - corresponding to zone $P D_{3}-T R_{1}$ in Fig. 10

Application of analytical methods is another option in order to find the bifurcation points. But this requires an application of an analytical method to get the 'slow flow' equations. Then, on the basis of nonlinear algebraic problem, we can find bifurcation points (see eg. [32,33]). However, due to additional assumptions, the method has also limitations. Therefore, we have decided to detect bifurcation points dealing directly with ODEs.

Of course, the continuation approach does not guarantee detection of all possible dynamical behaviour scenarios. Therefore, apart from the continuation method, a direct integration of the ODEs model has been applied for a large number of configuration parameters.

The computations have been repeated starting from different initial conditions. The reduced three degrees of freedom model result in $6 \mathrm{D}$ phase space which complicates the search of possible attractors. Thus, the computations have been limited varying the initial condition only for the first coordinate i.e. $q_{1}$ and equalling the rest to zero. The transient response has been discarded, and then, the solution has been plotted using stroboscopic projection, based on excitation frequency. The example of bifurcation scenario computed for $\Delta T=50$, seven selected initial conditions and $p_{1}$ treated as the bifurcation parameter is shown in Fig. 12. Apart from solutions obtained by a continuation method, we may observe much reacher dynamics represented by sequences of period doubling bifurcations. Around $p_{1}=3 \times 10^{-4}$, there is a cascade of period doubling leading to chaotic oscillations. This phenomenon is a result of both mechanical loading and increased nonuniformly distributed temperature.

The chaotic attractor plotted for $\Delta T=50$ and $p_{1}=3 \times 10^{-4}$ is presented in Fig. 13a. To prove that this chaotic behaviour results from both thermal and mechanical loading together, we computed a bifurcation diagram starting from chaotic attractor and then decreasing temperature, which is now treated as a bifurcation parameter. The final interval of the computations is shown in Fig. 13b. Starting from $\Delta T=47$ and varying temperature to $\Delta T=45$, we demonstrate transition from chaos to the regular triple point attractor.

\section{Conclusions and final remarks}

The dynamic behaviour of a geometrically nonlinear Timoshenko beam model under a nonuniformly distributed temperature and mechanical loadings is presented in the paper. The nonlinear model described by PDEs has been transformed to ODEs by the application of eigenmodes of linear Timoshenko beam. The correctness of the proposed reduced three-mode model has been verified by the FE method. A good agreement has been 


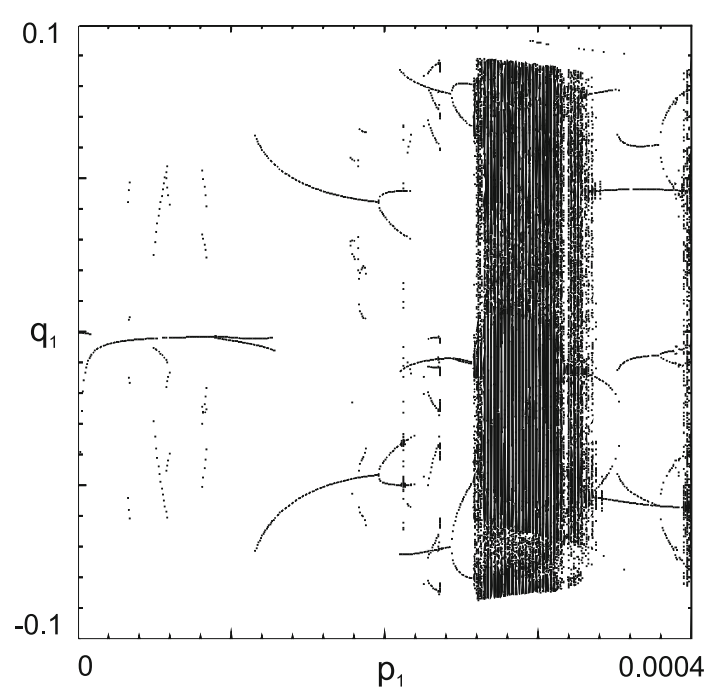

Fig. 12 Bifurcation diagrams versus amplitude of mechanical loading $p_{1}$ near the first resonance zone for temperature $\Delta T=50$ and fixed frequency $\Omega=0.029$; numerical integrations for different initial conditions

(a)

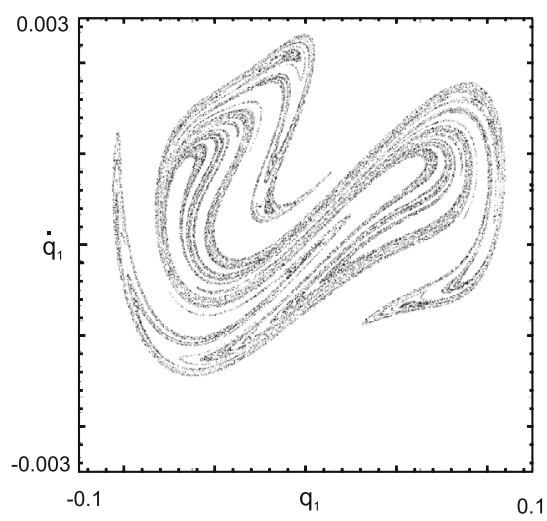

(b)

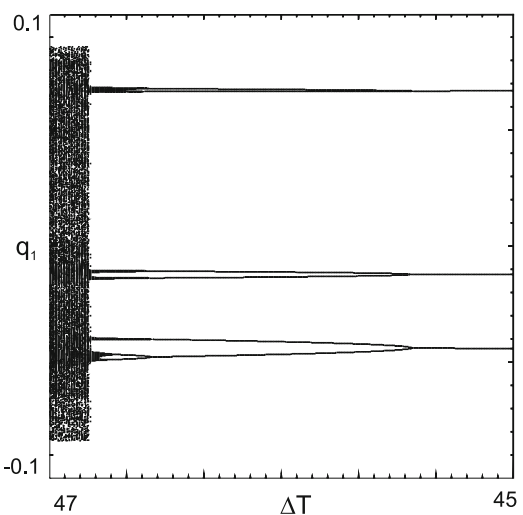

Fig. 13 Chaotic attractor (a) for $D T=50$ and bifurcation diagrams (b) versus thermal loading $\Delta T$ and fixed mechanical loading $p_{1}=3 \times 10^{-4}, \Omega=0.029$; bifurcation integrations started from the chaotic attractor

obtained for static tests under mechanical and as well as thermal loadings. Moreover, the eigenvalues from FE and analytical approach were consistent. Then, the detailed analysis of dynamics has been carried out near the first resonance zone considering an influence, either thermal or mechanical loadings. On the basis of analytical solutions, it has been shown that the system exhibited stiffening effect in the resonance curves for relatively large amplitude of excitation. The thermal loading, which has been nonuniformly distributed along the beam thickness and length, has a particular importance. The increase of the temperature difference $\Delta T$ leads to beam buckling, observed by a shift of the vibration centre. If the temperature difference is large, then the shift is essential, and furthermore, it affects the beam's natural frequencies. The nonlinear nature of thermal loading has been observed when mechanical loading increased. For large oscillations, thermal loading demonstrates nonlinear course (Fig. 6). Decreased temperature (having negative values) reduced bifurcation scenario and stabilised the solutions (Fig. 9). The increase of thermal loading, however, introduces additional instabilities, also for low mechanical loadings, leading to system's complex dynamics. The temperature distribution affects essentially beam response. If both thermal and mechanical loadings are large enough, then the period doubling and torus bifurcations occur which transit the beam into the chaotic oscillation region. The chaotic attractor located close to the bifurcation point can be suppressed, and then, the system moves to regular periodic oscillations, by a small decrease of temperature (Fig. 13). Varying temperature, we may switch the system between chaotic or regular attractors. It has been demonstrated in the paper that the level of thermal loading, as well as, its distribution must be considered as an important factor in real structural dynamics. 
Acknowledgments The second author wishes to acknowledge the partial support received from the Bulgarian NSF Grant DCVP 02/1. The third and fourth authors would like to acknowledge the financial support of Structural Funds in the Operational Programme Innovative Economy (IE OP) financed from the European Regional Development Fund Project Modern material technologies in aerospace industry, Nr POIG.01.01.02-00-015/08-00.

Open Access This article is distributed under the terms of the Creative Commons Attribution License which permits any use, distribution, and reproduction in any medium, provided the original author(s) and the source are credited.

\section{A Appendix: Integrals for longitudinal displacement with heat distribution}

Denoting $G_{u}^{A}=G_{u}+G_{u}^{T}$, the longitudinal displacement can be found from the first equation of the set (4). We find the integrals

$$
\int_{0}^{x} G_{u}^{A}(\xi) d \xi=-\left.\frac{1}{2}\left(\frac{\partial w}{\partial \xi}\right)^{2}\right|_{0} ^{x}+\frac{1}{2} T_{0} \Theta \alpha_{T} \sin \pi x
$$

and

$$
\int_{0}^{x} \xi G_{u}^{A}(\xi) d \xi=-\int_{0}^{x} \xi \frac{\partial w}{\partial \xi} \frac{\partial^{2} w}{\partial \xi^{2}} d \xi+\frac{T_{0} \Theta \alpha_{T}}{2 \pi}(-1+\cos \pi x+\pi x \sin \pi x)
$$

The above integrals can be computed in further steps by using the normal modes expansion for $w(x, t)$ displacement. Constant $K$ we can find from the boundary conditions of the studied particular problem. For a $S S-S S$ beam considered in this paper, the boundary conditions are $u(0)=u(1)=0$ which yields

$$
u(1)=\left[K+\int_{0}^{1} G_{u}^{A}(\xi) d \xi\right]-\int_{0}^{1} \xi G_{u}^{A}(\xi) d \xi=0
$$

and then we find

$$
\begin{aligned}
K & =-\int_{0}^{1} G_{u}^{A}(\xi) d \xi+\int_{0}^{1} \xi G_{u}^{A}(\xi) d \xi=\int_{0}^{1}(\xi-1) G_{u}^{A}(\xi) d \xi \\
& =\int_{0}^{1}(\xi-1) G_{u}(\xi) d \xi+\int_{0}^{1}(\xi-1) G_{u}^{T}(\xi) d \xi
\end{aligned}
$$

The second integral in Eq. (A.4) we can calculate easily considering the assumed heat transfer (Fig. 2)

$$
\int_{0}^{1}(\xi-1) G_{u}^{T}(\xi) d \xi=-\frac{T_{0} \Theta \alpha_{T}}{\pi}
$$

Taking into account the $G_{u}, G_{u}^{T}$ definitions, the first integral in Eq. (A.4) can be written in the form

$$
\int_{0}^{1}(\xi-1) G_{u}(\xi) d \xi=-\int_{0}^{1} \xi \frac{\partial w}{\partial \xi} \frac{\partial^{2} w}{\partial \xi^{2}} d \xi+\int_{0}^{1} \frac{\partial w}{\partial \xi} \frac{\partial^{2} w}{\partial \xi^{2}} d \xi
$$

For a symmetrically loaded beam

$$
\int_{0}^{1} \frac{\partial w}{\partial \xi} \frac{\partial^{2} w}{\partial \xi^{2}} d \xi=\left.\frac{1}{2}\left(\frac{\partial w}{\partial \xi}\right)^{2}\right|_{0} ^{1}=0
$$

and

$$
\int_{0}^{1} \xi \frac{\partial w}{\partial \xi} \frac{\partial^{2} w}{\partial \xi^{2}} d \xi=\frac{1}{2}\left[\left(\frac{\partial w}{\partial x}\right)_{x=1}^{2}-\int_{0}^{1}\left(\frac{\partial w}{\partial \xi}\right)^{2} d \xi\right]
$$

thus, we obtain the final form for the constant $K$

$$
K=-\frac{1}{2}\left[\left(\frac{\partial w}{\partial x}\right)_{x=1}^{2}-\int_{0}^{1}\left(\frac{\partial w}{\partial \xi}\right)^{2} d \xi\right]-\frac{T_{0} \Theta \alpha_{T}}{\pi}
$$


The function $G_{2}^{L}(x, t)$ we may express as

$$
G_{2}^{L}(x, t)=-\left[K+\int_{0}^{x} G_{u}^{A}(\xi) d \xi+\frac{1}{2}\left(\frac{\partial w}{\partial x}\right)^{2}\right] \frac{\partial^{2} w}{\partial x^{2}}
$$

\section{B Appendix: Galerkin Reduction}

The model represented by PDEs has been reduced to ODEs by means of Galerkin's orthogonalisation method. The generalised displacements vector is assumed in a series of a product of the quasi-normal modes (shape functions) $w_{n}(x), \psi_{n}(x)$ and generalised, time-dependent coordinates $q_{n}(t)$ as presented in Eq. (13). We assume $N_{f}$ as a number of taken modes. Substituting (13) into (12), we get

$$
\begin{aligned}
& \sum_{n=1}^{N_{f}}\left[\frac{d^{2} \psi_{n}}{d x^{2}}+\alpha \beta\left(\frac{d w_{n}}{d x}-\psi_{n}\right)\right] q_{n}(t)-d_{2} \sum_{n=1}^{N_{f}} \psi_{n} \dot{q}_{n}(t)-\sum_{n=1}^{N_{f}} \psi_{n} \ddot{q}_{n}(t)=G_{1}^{T} \\
& \beta \sum_{n=1}^{N_{f}}\left[\frac{d^{2} w_{n}}{d x^{2}}-\frac{d \psi_{n}}{d x}\right] q_{n}(t)-d_{1} \sum_{n=1}^{N_{f}} w_{n} \dot{q}_{n}(t)-\sum_{n} w_{n} \ddot{q}_{n}(t)=-p+G_{2}^{L}+G_{2}^{T}
\end{aligned}
$$

where $G_{1}^{T}$ is defined by (9b) and

$$
\begin{aligned}
G_{2}^{T} & =\frac{T_{0} \Theta \alpha_{T}}{2} \sin \pi x \sum_{n=1}^{N_{f}} q_{n} \frac{d^{2} w_{n}}{d x^{2}} \\
G_{2}^{L} & =-\left[-\int_{0}^{1} \sum_{n=1}^{N_{f}} \sum_{j=1}^{N_{f}} q_{n} \frac{d w_{n}}{d \xi} q_{j} \frac{d w_{j}}{d \xi} d \xi-T_{0} \Theta \alpha_{T}\left(\frac{1}{\pi}-\frac{1}{2} \sin \pi x\right)\right] \sum_{n=1}^{N_{f}} q_{n} \frac{d^{2} w_{n}}{d x^{2}}
\end{aligned}
$$

According to the Galerkin's procedure, the quasi-normal modes should satisfy geometrical and dynamical (natural) boundary conditions. For the reduction needs, we take linear modes of a $S S-S S$ beam. The eigenvalues and eigenmodes have been presented in details in the papers [29] and [30]. Considering that $w_{n}$ and $\psi_{n}$ are solutions of the eigenvalue problem, we obtain:

$$
\begin{aligned}
& \sum_{n=1}^{N_{f}}-\omega_{n}^{2} \psi_{n} q_{n}(t)-d_{2} \sum_{n=1}^{N_{f}} \psi_{n} \dot{q}_{n}(t)-\sum_{n=1}^{N_{f}} \psi_{n} \ddot{q}_{n}(t)=G_{1}^{T} \\
& \sum_{n=1}^{N_{f}}-\omega_{n}^{2} w_{n} q_{n}(t)-d_{1} \sum_{n=1}^{N_{f}} w_{n} \dot{q}_{n}(t)-\sum_{n=1}^{N_{f}} w_{n} \ddot{q}_{n}(t)=-p+G_{2}^{L}+G_{2}^{T}
\end{aligned}
$$

Multiplying (B.3a) by $\psi_{m}$, and (B.3b) by $w_{m}$, then summing up both equations, we have

$$
\begin{aligned}
& \sum_{n}^{N_{f}} \omega_{n}^{2}\left[\psi_{m} \psi_{n}+w_{m} w_{n}\right] q_{n}(t)+\sum_{n=1}^{N_{f}}\left(d_{2} \psi_{n} \psi_{m}+d_{1} w_{n} w_{m}\right) \dot{q}_{n}(t) \\
& +\sum_{n}^{N_{f}}\left[\psi_{m} \psi_{n}+w_{m} w_{n}\right] \ddot{q}_{n}(t)=-\psi_{m} G_{1}^{T}+w_{m}\left(p-G_{2}^{L}-G_{2}^{T}\right)
\end{aligned}
$$

Then, integrating (B.4) over the beam length, invoking the orthogonality condition [30],

$$
\int_{0}^{1}\left(\psi_{m} \psi_{n}+w_{m} w_{n}\right) d x= \begin{cases}1 & \text { for } n=m \\ 0 & \text { for } n \neq m\end{cases}
$$


and assuming proportional damping, the equations are transformed into the form:

$$
\begin{aligned}
& \ddot{q}_{n}(t)+2 \xi_{n} \omega_{n} \dot{q}(t)+\omega_{n}^{2} q_{n}(t) \\
& \quad=\int_{0}^{1} p(x, t) w_{n}(x) d x-\int_{0}^{1} G_{1}^{T}(x) \psi_{n}(x) d x-\int_{0}^{1} G_{2}^{L}(x, t) w_{n}(x) d x-\int_{0}^{1} G_{2}^{T}(x, t) w_{n}(x) d x
\end{aligned}
$$

where $\omega_{n}$ is the $n$-th natural frequency of the linear undamped Timoshenko beam and $\xi_{n}$ is a modal damping coefficient.

\section{Appendix: Coefficients of a reduced three modal beam with heat distribution}

The considered structure is a symmetric cross-ply laminated beam of length $L=0.5 \mathrm{~m}$ composed of 20 orthotropic layers, each $0.25 \mathrm{~mm}$ thick. The two layers located above and under the neutral surface have $0-90$ orientation with respect to $\mathrm{x}$-axis. The whole beam sequence of layers (plies) is $[(0 / 90) 8 / 0] s$. Material characteristics of the single ply are as follows:

Young's moduli: $E_{1}=56 \mathrm{GPa}, E_{2}=16 \mathrm{GPa}$,

Poisson's ratio: $v=0.269$,

thermal expansion coefficient: $\alpha_{T}=13.2 \times 10^{-6} \mathrm{~K}^{-1}$,

mass density $\rho=2,052 \mathrm{~kg} / \mathrm{m}^{3}$.

These characteristics correspond to a glass-epoxy composite material.

The effective Young modulus has been computed considering layers having $E_{1}$ with orientation 0 degrees and layers having $E_{2}$ with orientation 90 degrees. Using the simple summation formulae:

$$
\begin{aligned}
& A_{55}=b \sum_{k=1}^{N_{l}} G^{(k)}\left(z^{(k)}-z^{(k-1)}\right)=b \sum_{k=1}^{N_{l}} G^{(k)} h^{(k)} \\
& D=\frac{b}{3} \sum_{k=1}^{N_{l}} E^{(k)}\left(z^{(k)^{3}}-z^{(k-1)^{3}}\right), \quad \alpha_{T}=\frac{1}{N_{l}} \sum_{k=1}^{N_{l}} \alpha_{T}^{(k)}
\end{aligned}
$$

where $N_{l}=20$, we obtain the equations of composite Timoshenko beam in the form:

$$
\begin{aligned}
-\frac{\partial}{\partial x}\left(D \frac{\partial \psi}{\partial x}\right)+k^{s} A_{55}\left(\frac{\partial w}{\partial x}-\psi\right)-R I \frac{\partial^{2} \psi}{\partial t^{2}} & =0 \\
k^{s} \frac{\partial}{\partial x}\left[A_{55}\left(\frac{\partial w}{\partial x}-\psi\right)\right]+R H \frac{\partial^{2} w}{\partial t^{2}} & =-p
\end{aligned}
$$

To obtain $E_{e f}$ in the usual form of the Timoshenko beam, the coefficient D is divided by $\frac{b}{3} \sum_{k=1}^{N_{l}}\left(z^{(k)^{3}}-z^{(k-1)^{3}}\right)$.

The effective Young modulus of the beam is $E_{e f}=41.92 \mathrm{GPa}$, with natural frequencies of the beam

$$
\omega_{1}=40.9835 \mathrm{~Hz}, \omega_{2}=163.852 \mathrm{~Hz}, \omega_{3}=368.362 \mathrm{~Hz}
$$

Dimensionless natural frequencies take values

$$
\omega_{1}=0.0285, \omega_{2}=0.1139, \omega_{3}=0.256
$$

and coefficients

$$
\alpha=120,000 \quad \beta=0.328342
$$

mode 1

$$
\begin{aligned}
& C_{1,111}=0.412607, C_{1,112}=3.42356, C_{1,122}=0.443852, C_{1,123}=3.5799, \\
& C_{1,133}=0.451153, C_{1,113}=0, C_{1,0}^{T}=-1.39705 \times 10^{-7}, C_{1}^{T}=-3.81687 \times 10^{-6}
\end{aligned}
$$

mode 2

mode 3

$$
\begin{aligned}
& C_{2,222}=0.477462, \quad C_{2,122}=3.6828, \quad C_{2,223}=3.85098, \quad C_{2,211}=0.443852, \\
& C_{2,123}=0, \quad C_{2,233}=0.485317, \quad C_{2}^{T}=-4.1059 \times 10^{-6}
\end{aligned}
$$

$$
\begin{aligned}
& C_{3,333}=0.493301, C_{3,233}=3.91434, C_{3,133}=0, C_{3,113}=0.451153, \\
& C_{3,123}=3.74339, C_{3,223}=0.485317, C_{3}^{T}=-4.17345 \times 10^{-6}
\end{aligned}
$$




\section{References}

1. Warminski, J., Balthazar, J.M.: Nonlinear vibrations of a beam with a tip mass attached to a rotating hub. In: Proceedings of the ASME 2005, 20th Biennial Conference on Mechanical Vibration and Noise, vol. 1, pp. 1619-1624 (2005)

2. Hodges, D.H.: Review of composite rotor blade modeling. AIAA J. 28(3), 561-565 (1990)

3. Nayfeh, A.H., Balachandran, B.: Applied Nonlinear Dynamics: Analytical, Computational and Experimental Methods. Wiley, New York (1995)

4. Nayfeh, A.H., Pai, P.F.: Linear and Nonlinear Structural Mechanics. Wiley, New York (2004)

5. Lacarbonara, W.: Nonlinear Structural Mechanics. Springer, Berlin (2013)

6. Luongo, A., Zulli, D.: Mathematical Models of Beams and Cables. Wiley-ISTE, New York (2013)

7. Ranzi, G., Luongo, A.: A new approach for thin-walled member analysis in the framework of GBT. Thin Walled Struct. 49(11), 1404-1414 (2011)

8. Piccardo, G., Ranzi, G., Luongo, A.: A direct approach for the evaluation of the conventional modes within the GBT formulation. Thin Walled Struct. 74, 133-145 (2014)

9. Librescu, L., Song, O.: Thin-Walled Composite Beams: Theory and Application. Springer, Dordrecht (2006)

10. Mei, C., Decha-Umphai, K.: Finite element method for non-linear forced vibrations of beams. J. Sound Vib. 102(3), 369$380(1985)$

11. Gunda, J.B., Gupta, R.K., Janardhan, G.R., Rao, G.V.: Large amplitude free vibration analysis of Timoshenko beams using a relatively simple finite element formulation. Int. J. Mech. Sci. 52(12), 1597-1604 (2010)

12. Azrar, L., Benamar, R., White, R.G.: A semi-analytical approach to the non-linear dynamic response problem of S-S and $\mathrm{C}-\mathrm{C}$ beams at large vibration amplitudes, part I: General theory and application to the single mode approach to free and forced vibration analysis. J. Sound Vib. 224(2), 183-207 (1999)

13. Azrar, L., Benamar, R., White, R.G.: A semi-analytical approach to the non-linear, dynamic response problem of beams at large vibration amplitudes, part II: Multimode, approach to the steady state forced periodic response. J. Sound Vib. 255(1), 141 (2002)

14. Silvestre, N., Camotim, D.: First-order generalised beam theory for arbitrary orthotropic materials. Thin Walled Struct. 40, 755-789 (2002)

15. Ribeiro, P., Akhavan, H., Teter, A., Warminski, J.: A review on the mechanical behaviour of curvilinear fibre composite laminated panels. J. Compos. Mater (2014). doi:10.1177/0021998313502066

16. Boley, B.A., Weiner, J.H.: Theory of Thermal Stress. Wiley, New York (1960)

17. Nowacki, W.: Dynamic Problems of Thermoelasticity. Noordhoff, Leyden (1975)

18. Thorton, E.A.: Thermal Structures for Aerospace Applications, AIAA Education Series, Reston (1996)

19. Pradeep, V., Ganesan, N., Bhaskar, N.: Vibration and thermal buckling of composite sandwich beams with viscoelastic core. Compos. Struct. 87, 60-69 (2007)

20. Ganesan, N., Pradeep, V.: Buckling and vibration of sandwich beams with viscoelastic core under thermal environments. J. Sound Vib. 286, 1067-1074 (2005)

21. Treysséde, F.: Vibration analysis of horizontal self-weighted beams and cables with bending stiffness subjected to thermal loads. J. Sound Vib. 329, 1536-1552 (2010)

22. Treysséde, F.: Prebending effects upon the vibrational modes of thermally prestressed planar beams. J. Sound Vib. 307, 295$311(2007)$

23. Manoach, E., Ribeiro, P.: Coupled, thermoelastic, large amplitude vibrations of Timoshenko beams. Int. J. Mech. Sci. 46, 1589-1606 (2004)

24. Zhou, R.C., Xue, Y., Mei, C.: Finite element time domain-modal formulation for nonlinear flutter of composite panels. AIAA J. 32, 2044-2052 (1994)

25. Ribeiro, P., Manoach, E.: The effect of temperature on the large amplitude vibrations of curved beams. J. Sound Vib. 285, 1093-1107 (2005)

26. Manoach, E., Samborski, S., Mitura, A., Warminski, J.: Vibration based damage detection in composite beams under temperature variations using Poincare maps. Int. J. Mech. Sci. 62(1), 120-132 (2012)

27. Amabili, M., Carra, S.: Thermal effects on geometrically nonlinear vibrations of rectangular plates with fixed edges. J. Sound Vib. 321(2), 936-954 (2009)

28. Kazemirad, S., Ghayesh, M.H., Amabili, M.: Thermo-mechanical nonlinear dynamics of a buckled axially moving beam. Arch. Appl. Mech. 83(1), 25-42 (2013)

29. Warminska, A., Warminski, J., Manoach, E.: Temperature influence on nonlinear responses of Timoshenko beam. In: Proceedings of the ASME 2013 International Design Engineering Technical Conferences and Computers and Information in Engineering Conference, IDETC/CIE 2013, August 4-7, 2013, Portland, DETC2013-12624 (2013)

30. Warminska, A., Manoach, E., Warminski, J.: Nonlinear dynamics of a reduced multimodal Timoshenko beam subjected to thermal and mechanical loadings. Meccanica. (2014). doi:10.1007/s11012-014-9891-3

31. Warminska, A., Warminski, J., Manoach, E.: Thermal effects on internal and external resonances of a nonlinear Timoshenko beam. In: The ASME 2014 International Design Engineering Technical Conferences \& Computers and Information in Engineering Conference IDETC/CIE 2014, Buffalo, DETC2014-34078 (2014)

32. Luongo, A., Paolone, A., Di Egidio, A.: Qualitative analysis of classes of motion for multiresonant systems I. An algebraic method. Acta Mech. 174, 91-107 (2005)

33. Luongo, A., Paolone, A., Di Egidio, A.: Qualitative analysis of classes of motion for multiresonant systems II. A geometrical method. Acta Mech. 174, 109-124 (2005)

34. Friswell, M.I., Inman, D.J., Rietz, W.R.: Active damping of thermally induced vibrations. J. Intell. Mater. Syst. Struct. 8, 678685 (1997)

35. Han, S.H., Benaroya, H., Wei, T.: Dynamics of transversely vibrating beams using four engineering theories. J. Sound Vib. 225(5), 935-988 (1999) 
36. Abramovich, H., Elishakoff, I.: Influence of shear deformation and rotary inertia on vibration frequencies via Loves equation. J. Sound. Vib. 137, 516-522 (1990)

37. Hutchinson, J.R.: Transverse vibrations of beams, exact versus approximate solutions. J. Appl. Mech. 48, $923-928$ (1981)

38. Labuschagne, A., van Rensburg, N.F.J., van der Merweb, A.J.: Comparison of linear beam theories. Math. Comput. Model. 49, 20-30 (2009)

39. Hongzhi, Z., Qiang, G.: Nonlinear vibration analysis of Timoshenko beams using the differential quadrature method. Nonlinear Dyn. 32(3), 223-234 (2003)

40. Doedel, E.J., Champneys, A.R., Fairgrieve, T.F., Kuznetsov, Y.A., Sandstede, B., Wang, X.: Auto 97: continuation and bifurcation software for ordinary differential equations (1998). http://indy.cs.concordia.ca/auto 